\title{
TRANSPORT COEFFICIENTS IN PURE FLUIDS AND MIXTURES NEAR A CRITICAL POINT: COMPARISON OF THE ONE LOOP ORDER RESULTS WITH EXPERIMENT
}

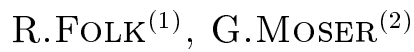 \\ (1) Institute for Theoretical Physics, \\ University of Linz, Austria \\ ${ }^{(2)}$ Institute for Physics and Biophysics, \\ University of Salzburg, Austria
}

Received September 12, 1995

\begin{abstract}
We review the nonasymptotic critical temperature dependence of the hydrodynamic transport coefficients in pure fluids near the critical point and in mixtures near the consolute point calculated in lowest nontrivial order of renormalization group theory. Two dynamical background parameters of the theory (respectively three for the mixtures) are fixed by fitting one of the transport coefficients (we take the shear viscosity). The other transport coefficents are then predicted without any adjustable parameter. Our analysis shows good agreement with the asymptotic one loop value of the Kawasaki amplitude $R=1.056$. Deviations of the transport coefficients in the asymptotic region are due to the one loop approximation for the asymptotic exponents.
\end{abstract}

\section{Introduction}

Universality of the dynamics at liquid and mixture second order phase transitions can be proven by measuring the asymptotic values of exponents and amplitude ratios of transport coefficients (TCs) calculated by renormalization group theory (RGT). In order to extract reliable values for these quantities it is necessary to include corrections to scaling in the analysis of the experimental data (for a review see [1]). This was interesting in itself since the correction amplitudes are also related by universal ratios. An extensive study of mixtures at the consolute point of this type has been performed in [2-4]. However it also has become clear that such a linearization may be insufficient in the experimental accessible region. The most prominent example for the indispensability of a nonlinear analysis, is the dynamics of ${ }^{4} \mathrm{He}$ near its superfluid phase transition [5] (for ${ }^{3} \mathrm{He}-{ }^{4} \mathrm{He}$ mixtures see [6]). A strategy for the treatment of nonuniversal behaviour by RGT was developed [7] which also applies for the pure liquid and mixture phase transitions. The main point of this strategy in our case is to describe the nonasymptotic behaviour of various physical quantities (e.g. TC's) by the flow of a small number of model parameters (e.g. mode couplings, ratios of model Onsager coefficients (OCs)). Once the flow of these parameters has been

(C) R.Folk, G.Moser, 1996

ISSN 0452-9910. Condensed Matter Physics 1996 No 7 (27-52) 
identified by fixing its initial conditions by a comparison with experiment, parameter free predictions are possible (e.g. a prediction of the temperature behaviour outside the region where experimental data are available, prediction of the temperature behaviour of TC's not used in the comparison with experiment). The strategy may lead even further, as has been shown for the superfluid transition, to the study of finite size effects [8], or the nonlinear-response regime [9] after a suitable extension of the theory.

The description of the crossover from background region (with regular nonuniversal behaviour) to the asymptotic region (with universal power law behaviour) requires the calculation of crossover functions which could be checked by comparison with experiment. For the second order phase transitions in pure liquids and mixtures (plait points, consolute points) most of such calculations were performed within mode coupling theory (MCT) (for a review see [10]) and decoupled mode theory [11,12]. Only recently such crossover functions have been calculated within RGT. In particular a nonasymptotic RGT of model $\mathrm{H}$ and $\mathrm{H}^{\prime}$ [13] has been formulated in $[14],[15],[16]$ and compared with experiments at the gas-liquid critical point in pure fluids as well as at the consolute point in mixtures [17] and at the plait point [14]. On the other hand important improvements have also been made within MCT by extending [18] an earlier version of MCT [19] (this formulation is based on the general theory of [20]) and comparing its theoretical results with experiments in pure fluids near the critical point [21] and mixtures near plait points [22].

RGT calculations are restricted to a certain region near the phase transition, usually of the order of $t=\left(T-T_{c}\right) / T_{c}<10^{-1}$ and do not treat the regular temperature behaviour. However within this region it treats the full TCs, whereas MCT considers the singular part only. The values of the TCs in the background are in fact the nonuniversal parameters within the RGT. The nonasymptotics is determined by the flow equations (these are systematically calculated in a loop expansion) of the model parameters. Universality is established by the flow of these parameters to their fixed point values, reached in the asymptotic region. Thus the exponents and amplitude ratios are not at our disposal, contrary to the MCT calculations, but fixed by the loop expansion. However it is promising that the calculated one loop RGT value for the Kawasaki amplitude (see equation below) is in agreement with the value adopted in MCT [21], [22] for optimal consistency with experiment.

The RGT calculation for the consolute point takes full account of the ratio $w$ (see equation (2.9) below) between the model H' OCs, so far neglected [13]. This model parameter may be found by comparison with the shear viscosity near the consolute point. However it appears most directly in the thermal conductivity at zero mass flow, which is finite at the consolute point, determining the magnitude of the critical enhancement. It would be very worthwhile to compare our theory with such measurements leading to much more accurate values of $w$.

\section{Pure fluids}

\subsection{Hydrodynamic transport coefficients}

The equations of motion of model $\mathrm{H}$ [13] for the order parameter $\phi_{0}\left(\phi_{0}=\right.$ $\sqrt{N_{A}} \sigma$ with $\sigma$ the entropy density per mass and $N_{A}$ the Avogadro number) 
and the transverse momentum density $\boldsymbol{j}_{t}$ are

$$
\begin{array}{r}
\frac{\partial \phi_{0}}{\partial t}=\stackrel{o}{\Gamma} \nabla^{2} \frac{\delta H}{\delta \phi_{0}}-\stackrel{o}{g}\left(\nabla \phi_{0}\right) \frac{\delta H}{\delta \boldsymbol{j}_{t}}+\Theta_{\phi}, \\
\frac{\partial \boldsymbol{j}_{t}}{\partial t}=\stackrel{o}{\lambda_{t}} \nabla^{2} \frac{\delta H}{\delta \boldsymbol{j}_{t}}+\mathcal{T}\left[\stackrel{o}{g}\left(\boldsymbol{\nabla} \phi_{0}\right) \frac{\delta H}{\delta \phi_{0}}\right]- \\
\stackrel{o}{g} \mathcal{T}\left\{\sum_{k}\left[j_{t, k} \nabla \frac{\delta H}{\delta j_{t, k}}-\nabla_{k} \boldsymbol{j}_{t} \frac{\delta H}{\delta j_{t, k}}\right]\right\}+\boldsymbol{\Theta}_{t} .
\end{array}
$$

where the Hamiltonian is given by

$$
H=\int d^{d} x\left\{\frac{1}{2} \stackrel{o}{r} \phi_{0}^{2}(x)+\frac{1}{2}\left(\nabla \phi_{0}(x)\right)^{2}+\frac{\stackrel{o}{u}}{4 !} \phi_{0}^{4}(x)+\frac{1}{2} a_{j} j_{t}^{2}(x)\right\}
$$

which contains the fourth order coupling $\stackrel{o}{u}$. The parameters in dynamics are the model-OCs $\stackrel{o}{\Gamma}, \stackrel{o}{\lambda} t$ and the mode coupling constant $\stackrel{o}{g}$. The fluctuating forces $\Theta_{t}$ and $\Theta_{\phi}$ fulfill the usual Einstein relations. The subscript ${ }_{0}$ at the densities and the superscript $o$ at the parameters indicate these quantities as unrenormalized.

The static order parameter correlations (inverse vertex function) are

$$
\left\langle\phi_{0} \phi_{0}\right\rangle_{c}=\frac{R T}{\rho}\left(\frac{\partial \sigma}{\partial T}\right)_{P}=\stackrel{o}{\Gamma}_{\phi \phi}^{-1} .
$$

Comparing the hydrodynamic limit within theory defined by the model equations (2.1)-(2.2) with the usual hydrodynamic equations for the entropy per mass $\sigma$ and the transverse momentum density $\boldsymbol{j}_{t}$ linearized in the velocity

$$
\frac{\partial \sigma}{\partial t}=\frac{\kappa}{\rho T} \nabla^{2} T, \quad \frac{\partial \boldsymbol{j}_{t}}{\partial t}=\frac{\bar{\eta}}{\rho} \nabla^{2} \boldsymbol{j}_{t}
$$

we find the identification of the thermal conductivity $\kappa$ and the shear viscosity $\bar{\eta}$ expressed by model vertex functions $\stackrel{o}{f}_{\alpha \tilde{\beta}}=\left.\frac{\partial}{\partial k^{2}} \stackrel{o}{\Gamma}_{\alpha \tilde{\beta}}\right|_{k=0}$ where $\alpha=\phi$ and $\tilde{\beta}=\tilde{\phi}$. This comparison gives for the dynamical coupling constant $\stackrel{o}{g}=R T / \sqrt{N_{A}}$ and the TCs

$$
\kappa=\rho T\left(\frac{\partial \sigma}{\partial T}\right)_{P} \stackrel{o}{f}_{\phi \tilde{\phi}}, \quad \bar{\eta}=\rho \stackrel{o}{f}_{t \tilde{t}} .
$$

The vertex functions $\stackrel{o}{f}_{\alpha \tilde{\beta}}$ appearing in (2.6) contain static contributions which can be separated according to a dissipation fluctuation theorem like $\stackrel{o}{f}_{\alpha \tilde{\beta}}=\stackrel{o}{\Gamma}_{\alpha \beta} \stackrel{o(d)}{f_{\alpha \tilde{\beta}}}$, leading to purely dynamic vertex functions $\stackrel{o}{f}_{\alpha \tilde{\beta}}^{(d)}$. Using the identification of the static vertex functions by thermodynamic derivatives one gets

$$
\stackrel{o}{f}_{\phi \tilde{\phi}}=\frac{\rho}{R T}\left(\frac{\partial T}{\partial \sigma}\right)_{P} \stackrel{o(d)}{f_{\phi \tilde{\phi}}^{(d)}}, \quad \stackrel{o}{f}_{t \tilde{t}}=a_{j} \stackrel{o}{f}_{t \tilde{t}}^{(d)}
$$


The purely dynamic vertex functions on the right hand side of the equations are in lowest order given by the unrenormalized OCs of model $\mathrm{H}$. A systematic loop expansion of the vertex functions leads to higher order contributions to the OCs. Inserting (2.7) into (2.6) the TCs simplify to

$$
\frac{\kappa}{\rho T}=\frac{\rho}{R T} \stackrel{o}{f}_{\phi \tilde{\phi}}^{o(d)}, \quad \frac{\bar{\eta}}{\rho}=a_{j}{\stackrel{o}{f_{t \tilde{t}}^{(d)}}}^{\prime}
$$

The dynamic vertex functions $\stackrel{o}{f}_{\alpha \tilde{\beta}}^{(d)}$ with $\alpha, \beta=\phi, t$ generally depend on the mode couplings and the OCs, this means $\stackrel{o}{f}_{\alpha \tilde{\beta}}^{(d)}=\stackrel{o(d)}{f_{\alpha \tilde{\beta}}}\left(\stackrel{o}{\Gamma}, \stackrel{o}{\lambda_{t}}, \stackrel{o}{g}, \stackrel{o o}{g} \stackrel{o}{u}\right)$. Performing the perturbation expansion in the usual way, one can see that the dynamic vertex functions are functions of ratios of the OCs and the mode couplings. Therefore we introduce suitable parameters

$$
\stackrel{o}{f}=\frac{\stackrel{o}{g}}{\sqrt{\stackrel{o}{\rho} \lambda_{t}}}, \quad \stackrel{o}{w_{\phi}}=\frac{\stackrel{o}{\Gamma}}{\stackrel{o}{\lambda}_{t}} .
$$

The Cut-Off dimensions of the original dynamic parameters are $[\stackrel{\circ}{\Gamma}]=0$ $\left[\lambda_{t}\right]=2$ and $[\stackrel{o}{g}]=1+\epsilon / 2(\epsilon=4-d)$. One can see that the ratio $\stackrel{o}{w}_{\phi}$ is irrelevant and will be set to zero.

\subsection{Renormalization}

The model will now be treated within the field theoretic renormalization group formalism $[23,24]$. We use the minimal subtraction scheme [25,26] where just the $\frac{1}{4-d}$ singularities in the vertex functions are absorbed in the $Z$-factors. The following static renormalizations are necessary

$$
\phi_{0}=Z_{\phi}^{1 / 2} \phi \quad \stackrel{o}{r}-\stackrel{o}{r}{ }_{s}=Z_{\phi}^{-1} Z_{r} r, \quad \stackrel{o}{u}=\mu^{\epsilon} Z_{\phi}^{-2} Z_{u} u A_{d}^{-1},
$$

where $\mu$ is a reference wave number, the factor $A_{d}=\Gamma(3-d / 2) /\left[2^{d-2} \pi^{d / 2}(d-\right.$ $2)]$. The renormalization of the static vertex functions reads

$$
\Gamma_{\phi \phi}=Z_{\phi} \stackrel{o}{\Gamma}_{\phi \phi} .
$$

From the conservation law follows that the $Z$-factors of the auxiliary fields $\tilde{\phi}_{0}$ which are defined analogous to $(2.10)$ are related to the renormalization factors of $\phi_{0}$ namely $Z_{\tilde{\phi}}=Z_{\phi}^{-1}$. Thus one has

$$
\tilde{\phi}_{0}=Z_{\phi}^{-1 / 2} \tilde{\phi} .
$$

The transverse momentum density $j$ does not renormalize, thus the corresponding conjugated field $\tilde{j}$ also does not renormalize. Using Ward identities which are a consequence of the Galilean invariance of the equations of motion [23] one finds, that the mode coupling $\stackrel{o}{g}$ needs no renormalization factor. We introduce the dimensionless coupling $g$

$$
\stackrel{o}{g}=\mu^{1+\epsilon / 2} g A_{d}^{-1 / 2} .
$$


The renormalized OCs are defined as

$$
\stackrel{o}{\Gamma}=Z_{\Gamma} \Gamma \quad, \quad \stackrel{o}{\lambda}=\mu^{2} Z_{\lambda_{t}} \lambda_{t} .
$$

From the factorization of the vertex functions into purely static and purely dynamic parts as discussed in the preceding section, it follows that the $Z_{\Gamma^{-}}$ factor also separate into purely static and purely dynamic parts, namely

$$
Z_{\Gamma}=Z_{\phi} Z_{\Gamma}^{(d)} .
$$

Using (2.10) and (2.12) the renormalized dynamic vertex functions are defined as

$$
\Gamma_{\phi \tilde{\phi}}=\stackrel{o}{\Gamma_{\phi \tilde{\phi}}}, \quad \Gamma_{t \tilde{t}}=\stackrel{o}{\Gamma_{t \tilde{t}}} .
$$

Inserting the separation of the static and dynamic parts of the $Z$-factors and the dynamic vertex functions the renormalization of the purely dynamic functions $f_{\alpha \tilde{\beta}}^{(d)}$ are obtained

$$
\stackrel{o^{(d)}}{f_{\phi \tilde{\phi}}}=Z_{\phi} f_{\phi \tilde{\phi}}^{(d)}, \quad \stackrel{o^{(d)}}{f_{t \tilde{t}}^{(d)}}=f_{t \tilde{t}}^{(d)} .
$$

The static vertex function of the transverse momentum density is simply given by the constant $a_{j}$. Therefore $Z_{\lambda_{t}}$ contains no static contributions. With (2.13) and (2.14) the relevant ratio defined in (2.9) renormalizes as

$$
\stackrel{o}{f}=\mu^{\epsilon / 2}\left(Z_{\Gamma} Z_{\lambda_{t}}\right)^{-1 / 2} f A_{d}^{-1 / 2} .
$$

The change of the renormalized parameters under renormalization is described by flow equations, which contain the $\zeta$-functions

$$
\zeta_{i}=\mu \frac{\partial \ln Z_{i}^{-1}}{\partial \mu}, \quad i=\phi, r, u, \Gamma, \lambda_{t} .
$$

The factorization of the renormalization constants in (2.15) leads to a separation of the corresponding $\zeta$-functions in static and purely dynamic parts. Inserting (2.15) in (2.19) one gets

$$
\zeta_{\Gamma}=\zeta_{\Gamma}^{(d)}+\zeta_{\phi} .
$$

The temperature dependence of the static and dynamic parameters is determined by the flow equations

$$
\begin{gathered}
\ell \frac{d u}{d \ell}=u\left(-\epsilon-2 \zeta_{\phi}[u(\ell)]+\zeta_{u}[u(\ell)]\right) \\
\ell \frac{d f}{d \ell}=-\frac{1}{2} f\left(\epsilon+\zeta_{\Gamma}^{(d)}[f(\ell), f(\ell) u(\ell)]+\zeta_{\lambda_{t}}[f(\ell), f(\ell) u(\ell)]+\zeta_{\phi}[u(\ell)]\right) \\
\ell \frac{d \Gamma}{d \ell}=\Gamma\left(\zeta_{\Gamma}^{(d)}[f(\ell), f(\ell) u(\ell)]+\zeta_{\phi}[u(\ell)]\right), \ell \frac{d \lambda_{t}}{d \ell}=\lambda_{t} \zeta_{\lambda_{t}}[f(\ell), f(\ell) u(\ell)] .
\end{gathered}
$$


The flow parameter $\ell$ is related to the relative temperature distance via the relation $[26]$

$$
\frac{r(\ell)}{(\mu \ell)^{2}}=1+\mathcal{O}\left(u^{2}\right), \quad r(\ell)=r(0) \exp \left[\int_{1}^{\ell} \frac{d x}{x}\left(\zeta_{r}-\zeta_{\phi}\right)\right] .
$$

The $\mathcal{O}(u(\ell))$-terms in the matching condition are found from the calculation of the correlation length $\xi$ [27] and ensure that with the choice $\mu=\xi_{0}^{-1}$ (in principle $\mu$ is a parameter within theory which at this stage of comparison we do not make use of; for the choice see remarks in [28] finally $\ell$ is related to $\xi$ by

$$
\ell=\xi_{0} \xi(t)^{-1}
$$

The fixed point relevant to critical behaviour is defined by the stable zero of the right hand sides of the flow equations. The resulting asymptotic power laws are governed by the exponents

$$
\nu=\frac{1}{2-\zeta_{r}^{*}+\zeta_{\phi}^{*}}, \quad \eta=-\zeta_{\phi}^{*}, \quad x_{\lambda}=-\zeta_{\Gamma}^{(d) *}, \quad x_{\eta}=-\zeta_{\lambda_{t}}^{*} .
$$

for the correlation length, the critical correlations at $T_{c}$, the thermal conductivity and the shear viscosity respectively (see Table [1] for the exponents used). The transient exponent $\omega_{f}$ is of the order $\mathcal{O}(1)$. The solution of the RGT-equation for the static vertex function is

$$
{\stackrel{o}{\Gamma_{\phi \phi}}}_{(u)}\left(\stackrel{o}{)}=(\mu \ell)^{2} Z_{\phi}^{-1} e^{\int_{1}^{\ell} \frac{d x}{x} \zeta_{\phi}} \hat{\Gamma}_{\phi \phi}[u(\ell)] .\right.
$$

The temperature dependence of the vertex functions (2.17) is determined by the corresponding renormalization group equations. Their solutions can be written as

$$
\begin{aligned}
& o_{\phi \tilde{\phi}}^{o(d)}(\{\Omega\})=Z_{\phi} e^{-\int_{1}^{\ell} \frac{d x}{x} \zeta_{\phi}} \hat{f}_{\phi \tilde{\phi}}^{(d)}[\{\Omega(\ell)\}], \\
& f_{t \tilde{t}}^{o(d)}(\{\Omega\})=(\mu \ell)^{2} \hat{f}_{t \tilde{t}}^{(d)}[\{\Omega(\ell)\}],
\end{aligned}
$$

where we have abbreviated the set $\left\{\Gamma, \lambda_{t}, g, g u\right\}$ by $\{\Omega\}$ and the $\ell$-dependent parameters correspondingly.

\subsection{Temperature dependence of the transport coefficients}

The temperature dependence of the TCs (2.6) is obtained by replacing the unrenormalized model parameters by the renormalized ones. Thus we have to insert equations (2.17) and (2.28) into (2.6). The temperature dependence of the renormalized static and dynamic parameters is governed by the flow equations (2.21) and (2.22). The connection between the flow parameter $\ell=\ell(t)$ and the temperature distance is given by $(2.25)$. The TCs then are

$$
\begin{gathered}
\frac{\kappa(\ell)}{\rho T}=\frac{\rho}{R T} Z_{\phi} e^{-\int_{1}^{\ell} \frac{d x}{x} \zeta_{\phi}} \Gamma(\ell)(1+G[f(\ell), f(\ell) u(\ell)]), \\
\bar{\eta}(\ell)=\frac{1}{R T}(\mu \ell)^{2} \lambda_{t}(\ell)(1+E[f(\ell), f(\ell) u(\ell)]) .
\end{gathered}
$$


The functions $G$ and $E$ represent the contributions from perturbation theory to $\hat{f}_{\phi \tilde{\phi}}^{(d)}$ and $\hat{f}_{t \tilde{t}}^{(d)}$. The corresponding one loop expressions can be found in (A.5).

For a calculation of the TCs as a function of the temperature it is convenient to replace the renormalization constants and exponential functions in (2.29) by the appropriate static quantities via the relations (2.27) and (2.4).

$$
\frac{\kappa(\ell)}{\rho T}=\left(\frac{\partial \sigma}{\partial T}\right)_{P}(\mu \ell)^{2} \hat{\Gamma}_{\phi \phi}[u(\ell)] \Gamma(\ell)(1+G[f(\ell), f(\ell) u(\ell)]) .
$$

The solutions of equations (2.23) can be rewritten as

$$
\Gamma(\ell)=\Gamma^{(d)}(\ell) e^{\int_{1}^{\ell} \frac{d x}{x} \zeta_{\phi}},
$$

where the dynamic part of the model OC $\Gamma^{(d)}$ is determined by the equation

$$
\ell \frac{d \Gamma^{(d)}}{d \ell}=\Gamma^{(d)} \zeta_{\Gamma}^{(d)}
$$

Thus we finally get eliminating $\ell$ by the matching condition $(2.25)$

$$
\kappa(t)=\rho C_{p}(t) \xi(t)^{-2} e^{\int_{1}^{\ell(t)} \frac{d x}{x} \zeta_{\phi}} \hat{\Gamma}_{\phi \phi}(u(t)) \Gamma^{(d)}(t)(1+G[f(t), f(t) u(t)]) .
$$

Using the relation $\left(A_{d}=\Gamma(3-d / 2) /\left[2^{d-2} \pi^{d / 2}(d-2)\right]\right)$

$$
\lambda_{t}(\ell)=A_{d} \frac{(R T)^{2}}{N_{A}} \frac{(\mu \ell)^{-3}}{e^{\int_{1}^{\ell} \frac{d x}{x} \zeta_{\phi}} \Gamma^{(d)}(\ell) f^{2}(\ell)},
$$

we can express the shear viscosity by $\Gamma^{(d)}(t)$ and $f(t)$

$$
\bar{\eta}(t)=A_{d} k_{B} T \xi(t) e^{-\int_{1}^{\ell(t)} \frac{d x}{x} \zeta_{\phi}(u(x))} \frac{1+E[f(t), f(t) u(t)]}{\Gamma^{(d)}(t) f^{2}(t)} .
$$

equations (2.34) and (2.36) constitute the main result of RGT for the TCs in a pure liquid. One may further simplify the expressions by approximating $\zeta_{\phi}(u(t))$ by its asymptotic value $-\eta$ as well as the static amplitude function. Then

$$
\kappa(t)=\rho C_{p}(t) \xi_{0}^{-\eta} \xi(t)^{-2+\eta} \hat{\Gamma}_{\phi \phi}\left(u^{*}\right) \Gamma^{(d)}(t)(1+G[f(t), f(t) u(t)]) .
$$

or the thermal diffusivity $D_{T}=\kappa /\left(\rho C_{p}\right)$

$$
D_{T}(t)=\xi_{0}^{-\eta} \xi(t)^{-2+\eta} \hat{\Gamma}_{\phi \phi}\left(u^{*}\right) \Gamma^{(d)}(t)(1+G[f(t), f(t) u(t)])
$$

and

$$
\bar{\eta}(t)=A_{d} k_{B} T \xi_{0}^{\eta} \xi(t)^{\epsilon-\eta} \frac{1+E[f(t), f(t) u(t)]}{\Gamma^{(d)}(t) f^{2}(t)} .
$$

The static amplitude function $\hat{\Gamma}_{\phi \phi}$ in one loop order is one. In principle the temperature dependence of the static coupling $u(\ell(t))$ can be found 
from the specific heat [29]. At every place where possible we replace the static theoretical quantities by their experimental counterpart. The temperature dependent dynamic parameter $f(\ell(t))$ are obtained by solving the flow equations $(2.22)$. The remaining unknown quantities are the initial values $\Gamma^{(d)}\left(t_{0}\right)$ and $f\left(t_{0}\right)$. These are found by fitting one of the TCs (2.6) or (2.36) over a certain temperature interval to experimental data.

Asymptotically when the dynamical parameters take their fixed point values we get the following behaviour of the TCs with temperature (at $d=3)$

$$
\kappa=\kappa_{a} t^{-x_{\lambda} \nu}, \quad D_{T}=D_{a T} t^{\left(1+x_{\eta}\right) \nu}, \quad \bar{\eta}=\bar{\eta}_{a} t^{-x_{\eta} \nu} .
$$

We have made use of the asymptotic behaviour of the static quantities

$$
\rho C_{p}=\chi_{a} t^{-\gamma}, \quad \xi=\xi_{0} t^{-\nu} .
$$

and the scaling law $\gamma=(2-\eta) \nu$ and $x_{\lambda}+x_{\eta}=\epsilon-\eta$.

\subsection{Nonuniversal Kawasaki amplitude}

The Kawasaki amplitude is defined (at $d=3$ relative to its mode coupling value $\left.\frac{1}{6 \pi}\right)$ as $[30]$

$$
R_{e x p}^{\text {pure }}=\frac{6 \pi \bar{\eta} \kappa \xi}{\rho k_{B} T^{2}}\left(\frac{\partial T}{\partial \sigma}\right)_{P} .
$$

Inserting the theoretical expressions we obtain the nonasymptotic amplitude for $d=3\left(A_{3}=\frac{1}{4 \pi}\right)$

$$
R_{t h}(\ell)=\frac{3}{2} \hat{\Gamma}_{\phi \phi}(u(\ell)) \frac{(1+G[f(\ell), f(\ell) u(\ell)])(1+E[f(\ell), f(\ell) u(\ell)])}{f^{2}(\ell)} .
$$

At $T=T_{c}$ it is expected that $R_{e x p}^{\text {pure }}$ reaches its universal value $[13,23,24,15]$ $R_{\text {theor }}^{*}$ (see Table 1 ) which reads

$$
R_{\text {theor }}^{*}=\frac{3}{2} \hat{\Gamma}_{\phi \phi}\left(u^{*}\right) \frac{\left(1+G\left[f^{*}, f^{*} u^{*}\right]\right)\left(1+E\left[f^{*}, f^{*} u^{*}\right]\right)}{f^{* 2}} .
$$

\begin{tabular}{|c|c|c|c|c|c|c|}
\hline Ref. & $\nu$ & $\eta$ & $f^{2 *}$ & $x_{\lambda}$ & $x_{\eta}$ & $R$ \\
\hline this work & 0.63 & 0 & 1.12 & 0.95 & 0.05 & 1.056 \\
two loop & 0.69 & 0.019 & $1.02[13]$ & $0.908[13]$ & $0.073[13]$ & \\
{$[21]$} & 0.63 & 0.033 & - & 0.904 & 0.063 & 1.05 \\
\hline
\end{tabular}

Table 1. Values used in the comparison with experiment

On the other hand inserting the asymptotic behaviour of the quantities involved into the experimental expression we obtain

$$
R_{e x p}^{*}=\frac{6 \pi \bar{\eta}_{a} \kappa_{a} \xi_{0}}{k_{B} T_{c} \chi_{a}}
$$

or if the thermal diffusivity is measured

$$
R_{e x p}^{\text {pure }}=\frac{6 \pi \bar{\eta} D_{T} \xi}{k_{B} T}, \quad R_{e x p}^{*}=\frac{6 \pi \bar{\eta}_{a} D_{T a} \xi_{0}}{k_{B} T_{c}} .
$$




\subsection{Expected temperature behaviour according to the theory}

The temperature dependence of the dynamical part of the order parameter OC, equations $(2.23,2.33)$, the shear viscosity, equation (2.39), and the thermal conductivity or diffusivity, equation (2.37) or equation (2.38), and the Kawasaki amplitude, equation (2.42), depend on the flow of the dynamical parameter $f$ equation (2.22). All these quantities reach finite background values at temperatures depending on the temperature where the correlation length itself reaches its background value. This value defines the value of $\ell$ (via the matching condition equation (2.25)), at which the flow of all parameters becomes stationary. figure 1 demonstrates this for an theoretical example. We have used the simple temperature dependence of the correlation length $\xi(t)=\xi_{0} t^{\nu}+\xi_{b}$. Note that the observable value of the crossover temperature to a constant background in the OC and the TC's also depends on the crossover temperature in the flow of the model parameter $f$ (which depends on the background value and the flow equation) and on the dependence of the OC and TC's on the parameter $f$.

\section{Comparison with experiment in pure fluids}

\subsection{General procedure}

Our analysis of the experimental data, quite similar to the general strategy developed for the superfluid transition [7] is the following:

(i) We determine the parameters $\Gamma^{(d)}\left(t_{0}\right)$ and $f\left(t_{0}\right)$ by a fit of the shear viscosity in the temperature region $t_{1} \leq t \leq t_{0}$. We prefer to take the shear viscosity since there are no static quantities involved besides the correlation length and since $f$ enters in leading order, which makes it more sensitive to the flow of $f$. For the correlation length we take, for the lack of anything better, the asymptotic power law $\xi=\xi_{0} t^{-\nu}$ (values are taken from the Table 4.3.4 in [31]), which is assumed to be valid in most of the cases in the region of the fit. It would be very desirable to have more experimental information on $\xi$ especially in the crossover region to its background value.

As discussed in the preceding section the theoretical temperature dependence leads to a temperature independent background behaviour of the shear viscosity. However the experimental shear viscosity has a a regular temperature dependence in the background not contained in the RGT. Therfore if one extends the region of fit into the region where the regular temperature dependence sets in one has to correct for that dependence. This can be done in the following simple way. From a fit of the shear viscosity outside the critical region $\left(t>t_{r e g}\right)$ one determines the regular part of the shear viscosity $\bar{\eta}_{r e g}(t)$. This regular part takes the value $\bar{\eta}_{r e g}(0)$ at $T_{c}$. Then the corrected experimental shear viscosity corresponding to the shear viscosity calculated by RGT is

$$
\bar{\eta}_{\text {corr }}(t)=\bar{\eta}_{e x p}(t)-\bar{\eta}_{\text {reg }}(t)+\bar{\eta}_{\text {reg }}(0) .
$$

In mode coupling theory the procedure in the comparison is somewhat different since there the complete background shear viscosity has to be subtracted in order to compare only the singular part of the shear viscosity with theory (see figure 2 in [10]). Extending the fit region into the region of constant background values is only possible if the temperature dependence of the correlation length over the whole region is known. For the examples of pure fluids discussed below we restricted our fit to a region, where such 


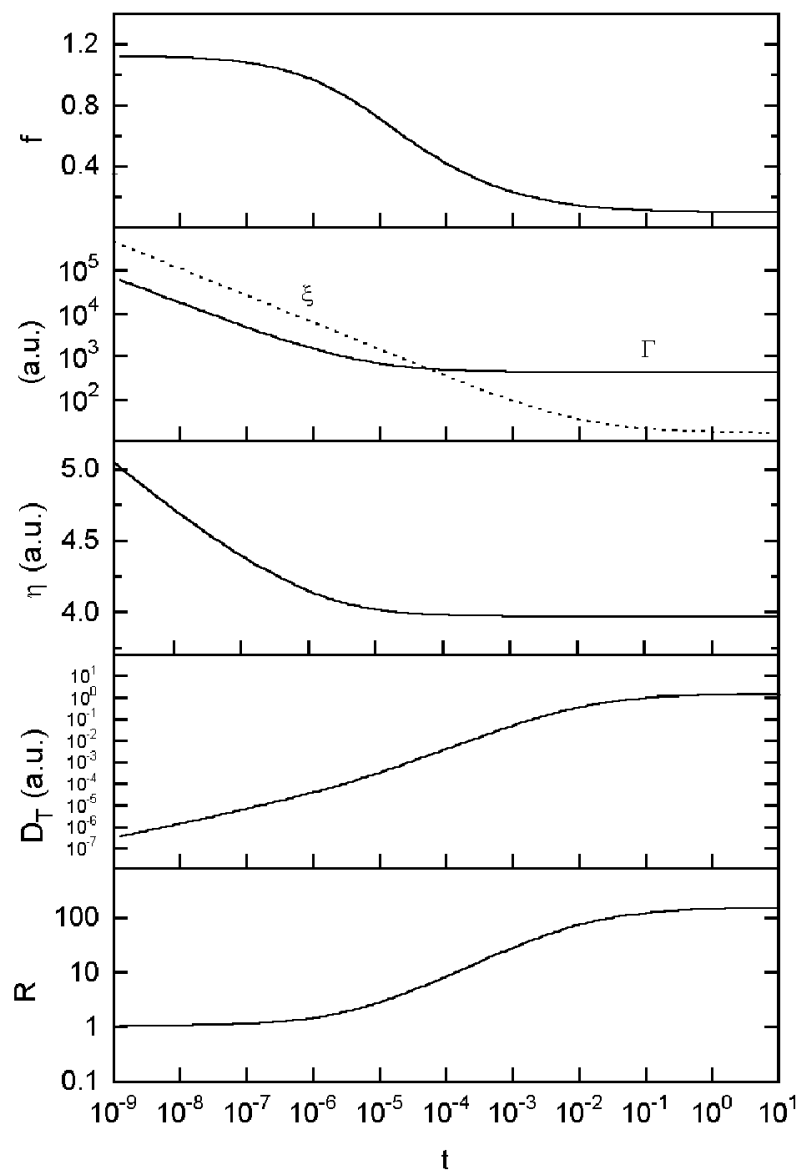

Figure 1. Temperature dependence of (a) the mode-coupling $f$ according to the flow equation (2.22), (b) the correlation length $\xi$ and the order parameter diffusion rate $\Gamma$ according to the flow equation $(2.23)$, (c) the shear viscosity $\bar{\eta}$ according to equation (2.39), (d) the thermal diffusivity $D$ according to equation (2.38), and the (e) Kawasaki amplitude ratio $R$ according to equation (2.46) in one loop order (Appendix A). All quantities reach temperature independent background values, $f$ and $R$ reach at $T_{c}$ their one loop fixed point values $f^{*}$ and $R^{*}=1.056$. 
correction could be neglected, however for the mixtures discussed below we demonstrate, in one example, the effects of considering $\bar{\eta}_{c o r r}$ instead of $\bar{\eta}_{\text {exp }}$.

(ii) We calculate the thermal conductivity or thermal diffusivity. In the last case the result is fixed by (i) alone. Otherwise the prediction of the thermal conductivity depends on the data of $\rho C_{p}$ which has to to be calculated via the thermodynamic relation

$$
\rho C_{p}=\rho C_{v}+T\left(\frac{d P}{d T}\right)_{\rho}^{2}\left(\frac{d \rho}{d P}\right)_{T} .
$$

Thus several static measurement are involved and enter the quality of the prediction.

The measurements are effected by gravity near $T_{c}$ and by the regular temperature dependence further a way from $T_{c}$. Thus without any corrections the analysis works within a temperature window $t_{g}<t<t_{\text {reg }}$. Of course these border line temperatures depend on the specific liquid, typical values of $t_{\text {reg }}$ are $10^{-1}$ to $10^{-2}$ and apart of $\mathrm{He} t_{g}$ is smaller than $10^{-4}$ for $\mathrm{CO}_{2}$ even smaller than $10^{-7}[31]$.

Comparison with ${ }^{3} \mathrm{He}$ As already indicated we take the experimental results for the static quantities. These are represented by power laws in the experimental temperature region $10^{-3} \leq t \leq 10^{-1}$. We use the following representations of experimental data common in literature

$$
\begin{aligned}
\left(\frac{d \rho}{d P}\right)_{T} & =\Gamma_{T} t^{-\gamma} \\
\left(\frac{d P}{d T}\right)_{\rho} & =a_{0}+a_{1} t+a_{2} t^{2}+A t^{1-\alpha} \\
C_{v} & =t^{-\alpha}\left(A_{1}+A_{2} t^{\Delta}\right) .
\end{aligned}
$$

The parameters used for ${ }^{3} \mathrm{He}$ and ${ }^{4} \mathrm{He}$ are listed in Table 2. From the

\begin{tabular}{|c|c|c|}
\hline & ${ }^{3} \mathrm{He}$ & ${ }^{4} \mathrm{He}$ \\
\hline$\rho\left[\mathrm{g} / \mathrm{cm}^{3}\right]$ & 0.04145 & 0.0696 \\
$\gamma$ & 1.19 & 1.19 \\
$\alpha$ & 0.11 & 0.11 \\
$\Delta$ & 0.54 & 0.54 \\
$\Gamma_{T} 10^{4}\left[\mathrm{~g} /\left(\mathrm{cm}^{3}\right.\right.$ Torr $\left.)\right]$ & 2.23 & 0.753 \\
$a_{0}[$ Torr $/ \mathrm{K}]$ & 888.0 & 1289.2 \\
$a_{1}[$ Torr $/ \mathrm{K}]$ & -1294.14 & -5190.46 \\
$a_{2}[$ Torr $/ \mathrm{K}]$ & 0 & 4504.99 \\
$A[$ Torr $/ \mathrm{K}]$ & 1288.98 & 4085.66 \\
$A_{1}[\mathrm{~J} /(\mathrm{moleK})]$ & 25.11 & 48.00 \\
$A_{2}[\mathrm{~J} /($ moleK $)]$ & 2.6 & 2.6 \\
\hline
\end{tabular}

Table 2. Parameters found from fits of the static quantities with the functions in equations (49) for liquid ${ }^{3} \mathrm{He}$ and ${ }^{4} \mathrm{He}$.

measurements of $\left(\frac{d \rho}{d P}\right)_{T}[32],\left(\frac{d P}{d T}\right)_{\rho}[33]$, both are strongly diverging susceptibilities, and $C_{v}$ [34] an effective power law for $\rho C_{p}$ holds over that temperature region. 
First we fit the shear viscosity data [35] by equation (2.36) with the initial conditions $\Gamma\left(t=10^{-1}\right)$ and $f\left(t=10^{-1}\right)$ as fit parameters and find the flow of these parameters (see figure 2 ). Then we predict without any further parameter the thermal conductivity by equation (2.34) and compare with the thermal conductivity of [36] and the Kawasaki amplitude (the values are found by interpolating the shear viscosity data) (see again figure 2).

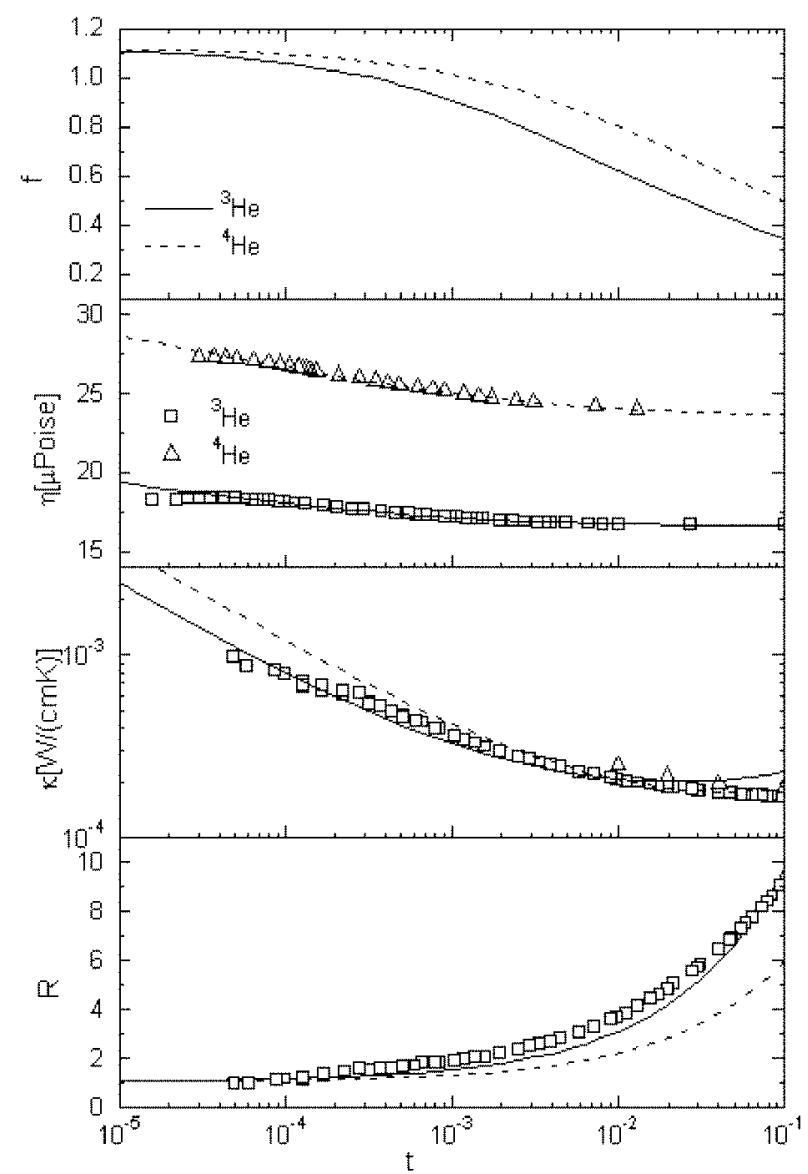

Figure 2. Comparison of theory with ${ }^{3} \mathrm{He}$ and ${ }^{4} \mathrm{He}$ data (see text for the Refs.). We show the flow of $f$ found from a fit of the shear viscosity $\eta$ and compare with the prediction of the thermal conductivity $\kappa$. We also compare with the Kawasaki amplitude $R$ for ${ }^{3} \mathrm{He}$, and give the prediction for ${ }^{4} \mathrm{He}$.

Comparison with ${ }^{4} \mathrm{He}$ The experimental situation in ${ }^{4} \mathrm{He}$ is not so favourable as in ${ }^{3} \mathrm{He}$ since there are not enough dynamical data for the thermal conductivity [37] and there are less exact static data. Nevertheless we want to see if qualitative agreement can be achieved. Again from the measurements of $\left(\frac{d \rho}{d P}\right)_{T}[38],\left(\frac{d P}{d T}\right)_{\rho}[39]$ and $C_{v}[40]$ we determine $\rho C_{p}$. 
The correlation length has been measured in light scattering experiments [41] and the values of $\xi_{0}=2.2 \pm 0.6 \AA$ and $\nu=0.62 \pm 0.1$ have been found together with the same value of $\gamma$ as in the compressibility measurements [38]. Not that although the value of $\nu$ is compatible with the hyperscaling law, it leads to $\nu=2-\frac{\gamma}{\nu}=0.13$ which is much larger than the expected value $\eta=0.033[10]$. The same analysis before leads to the results shown in figure 2 .

Comparison with Ethane The shear viscosity has been measured by [42], the thermal conductivity in the critical region by Mostert [43], and the thermal diffusivity in [44]. Thus from statics we only need the correlation length again taken in its asymptotic form. The same quality for our fit and for the prediction is reached as in the case of He.

All three analyzed cases show a considerable temperature dependence of the model parameters (see e.g. $f(t)$ ) in the experimental region which reflects itself in the strong temperature dependence of the Kawasaki amplitude. However since there are no experimental data of the correlation length into the region of constant $\xi$, we cannot extend our analysis of the TC's into the regular region. Therefore the thermal diffusivity in this region shows no indication of the onset of the regular region (a bending over as seen in the theoretical curve of figure 1).

We just mention that we have used the flow found from the fit of the shear viscosity to compare our prediction of the sound attenuation (see [17]) with the data of [45] for ${ }^{3} \mathrm{He}$ and ${ }^{4} \mathrm{He}$ and also good agreement has been found. Preliminary application of this analysis to other pure liquids lead to similar conclusions.

\section{Mixtures near the consolute point}

\subsection{Hydrodynamic transport coefficients}

In the critical dynamics of a mixture we have to consider in addition to the densities of the pure fluid case the local concentration $c(x)$. In fact it turns out that at the consolute point the local concentration constitutes the order parameter density,

$$
\begin{aligned}
\phi_{0}(x) & =\sqrt{N_{A}}(\triangle c(x)-\langle\triangle c(x)\rangle), \\
q_{0}(x) & =\sqrt{N_{A}}\left[\triangle \sigma(x)-\left(\frac{\partial \sigma}{\partial c}\right)_{T, P}(\triangle c(x)-\langle\triangle c(x)\rangle)\right] .
\end{aligned}
$$

The static Hamiltonian then reads

$$
\begin{aligned}
H= & \int d^{d} x\left\{\frac{1}{2} \stackrel{o}{\mathcal{T}} \phi_{0}^{2}(x)+\frac{1}{2}\left(\nabla \phi_{0}(x)\right)^{2}+\frac{\stackrel{o}{\tilde{u}}}{4 !} \phi_{0}^{4}(x)\right. \\
& \left.+\frac{1}{2} a_{q} q_{0}^{2}(x)+\frac{1}{2} \stackrel{o}{\gamma}_{q} q_{0}(x) \phi_{0}^{2}(x)+\frac{1}{2} a_{j} \boldsymbol{j}_{t}^{2}(x)\right\} .
\end{aligned}
$$

with the static correlations

$$
\langle\phi \phi\rangle_{c}=\frac{R T}{\rho}\left(\frac{\partial c}{\partial \Delta}\right)_{T, P},
$$




$$
\langle q q\rangle_{c}=\frac{R T}{\rho}\left(\frac{\partial \sigma}{\partial T}\right)_{c, P} .
$$

There are now two critical susceptibilities, a strong one (4.3) diverging with exponent $\gamma$ and a weak one (4.4) diverging with exponent $\alpha$.

The set of appropriate dynamic equations are those of model H' [13]

$$
\begin{gathered}
\frac{\partial \phi_{0}}{\partial t}=\stackrel{o}{\Gamma} \nabla^{2} \frac{\delta H}{\delta \phi_{0}}+\stackrel{o}{L} \nabla^{2} \frac{\delta H}{\delta q_{0}}-\stackrel{o}{g}\left(\nabla \phi_{0}\right) \frac{\delta H}{\delta \boldsymbol{j}_{t}}+\Theta_{\phi}, \\
\frac{\partial q_{0}}{\partial t}=\stackrel{o}{L} \nabla^{2} \frac{\delta H}{\delta \phi_{0}}+\stackrel{o}{\lambda_{q}} \nabla^{2} \frac{\delta H}{\delta q_{0}}-\stackrel{o}{g}\left(\nabla q_{0}\right) \frac{\delta H}{\delta \boldsymbol{j}_{t}}+\Theta_{q}, \\
\frac{\partial \boldsymbol{j}_{t}}{\partial t}=\stackrel{o}{\lambda_{t}} \nabla^{2} \frac{\delta H}{\delta \boldsymbol{j}_{t}}+\mathcal{T}\left[\stackrel{o}{g}\left(\nabla \phi_{0}\right) \frac{\delta H}{\delta \phi_{0}}+\stackrel{o}{g}\left(\nabla q_{0}\right) \frac{\delta H}{\delta q_{0}}\right] \\
-\stackrel{o}{g} \mathcal{T}\left\{\sum_{k}\left[j_{t, k} \nabla \frac{\delta H}{\delta j_{t, k}}-\nabla_{k} \boldsymbol{j}_{t} \frac{\delta H}{\delta j_{t, k}}\right]\right\}+\boldsymbol{\Theta}_{t} .
\end{gathered}
$$

There are now three model OCs according to the modes of mass diffusion, heat conduction and the modes of heat transport induced by a concentration difference or mass transport induced by a temperature difference. One may proceed as in the case of the pure fluid and compare with the hydrodynamic equations for a mixture

$$
\begin{gathered}
\frac{\partial \sigma}{\partial t}=\left[\frac{D k_{T}}{T}\left(\frac{k_{T}}{T}\left(\frac{\partial \Delta}{\partial c}\right)_{T, P}-\left(\frac{\partial \Delta}{\partial T}\right)_{c, P}\right)+\frac{\kappa}{\rho T}\right] \nabla^{2} T \\
+D\left[\frac{k_{T}}{T}\left(\frac{\partial \Delta}{\partial c}\right)_{T, P}-\left(\frac{\partial \Delta}{\partial T}\right)_{c, P}\right] \nabla^{2} c \\
\frac{\partial c}{\partial t}=\frac{D k_{T}}{T} \nabla^{2} T+D \nabla^{2} c \\
\frac{\partial \boldsymbol{j}_{t}}{\partial t}=\frac{\eta}{\rho} \nabla^{2} \boldsymbol{j}_{t}
\end{gathered}
$$

in order to identify the physical TC's by the model vertex functions

$$
\begin{gathered}
D=\stackrel{o}{f_{\phi \tilde{\phi}},} \\
\frac{k_{T}}{T}=\frac{1}{D}\left(\frac{\partial \sigma}{\partial T}\right)_{c, P} \stackrel{o}{f}_{q \tilde{\phi}}, \\
\frac{\kappa}{\rho T}=\left(\frac{\partial \sigma}{\partial T}\right)_{c, P} \frac{1}{D}\left[\stackrel{o}{\left.f_{\phi \tilde{\phi}} f_{q \tilde{q}}-\stackrel{o}{f}_{q \tilde{\phi}} f_{\phi \tilde{q}}^{o}\right] .}\right.
\end{gathered}
$$

Separating the static parts from the vertex functions

$$
\stackrel{o}{f}_{\phi \tilde{\phi}}=\frac{\rho}{R T}\left(\frac{\partial \Delta}{\partial c}\right)_{T, P} \stackrel{o(d)}{f_{\phi \tilde{\phi}}^{(d)}}, \quad \stackrel{o}{f}_{\phi \tilde{q}}=\frac{\rho}{R T}\left(\frac{\partial \Delta}{\partial c}\right)_{T, P} \stackrel{o(d)}{f}_{\phi \tilde{q}}^{(d)},
$$




$$
\stackrel{o}{f_{q \tilde{\phi}}}=\frac{\rho}{R T}\left(\frac{\partial T}{\partial \sigma}\right)_{c, P} \stackrel{o(d)}{f_{q \tilde{\phi}}}, \quad \stackrel{o}{f}_{q \tilde{q}}=\frac{\rho}{R T}\left(\frac{\partial T}{\partial \sigma}\right)_{c, P} \stackrel{o(d)}{f_{q \tilde{q}}^{(d)}},
$$

we get

$$
\begin{gathered}
D=\frac{\rho}{R T}\left(\frac{\partial \Delta}{\partial c}\right)_{T, P} \underset{f_{\phi \tilde{\phi}}^{o(d)}}{f^{(d)}}, \\
\frac{k_{T}}{T}=\frac{\rho}{R T} \frac{1}{D} \stackrel{o(d)}{f}_{q \tilde{\phi}}, \\
\frac{\kappa}{\rho T}=\left(\frac{\rho}{R T}\right)^{2}\left(\frac{\partial \Delta}{\partial c}\right)_{T, P} \frac{1}{D}\left[\stackrel{o}{f}_{\phi \tilde{\phi}}^{(d)} f_{q \tilde{q}}^{(d)}-\left(\stackrel{o(d)}{f_{q \tilde{\phi}}^{(d)}}\right)^{2}\right] .
\end{gathered}
$$

Note that the thermal conductivity is defined under the condition of zero mass flow. Since $\stackrel{o(d)}{f} f_{q \tilde{\phi}}=\stackrel{o}{L}$ we have the relation $k_{T} D=\frac{\rho}{\rho}$.

We again define suitable dynamical parameters

$$
\begin{gathered}
\stackrel{o}{w}=\frac{\stackrel{o}{L}}{\sqrt{\frac{o}{\Gamma} \lambda_{q}^{o}}}, \quad \stackrel{o}{f}=\frac{\stackrel{o}{g}}{\sqrt{\frac{o o}{\Gamma} \lambda_{t}}}, \\
\stackrel{o}{w_{q}}=\frac{\stackrel{o}{\lambda_{q}}}{\stackrel{o}{\lambda_{t}}}, \quad \stackrel{o}{w_{\phi}}=\frac{\stackrel{o}{\Gamma}}{\stackrel{o}{\lambda}_{t}}, \quad \stackrel{o}{\boldsymbol{w}_{4}}=\frac{\stackrel{o}{\Gamma}}{\lambda_{q}} .
\end{gathered}
$$

The Cut-Off dimensions of the original dynamic parameters are $[\stackrel{\circ}{\Gamma}]=0$, $[\stackrel{o}{L}]=1,\left[\stackrel{o}{\lambda_{q}}\right]=\left[\stackrel{o}{\lambda}_{t}\right]=2$ and $[\stackrel{o}{g}]=1+\epsilon / 2(\epsilon=4-d)$. One can see that the ratios in (4.19) have dimension zero, while in (4.20) the dimensions are negative $\left(\left[\stackrel{o}{w}_{\phi}\right]=\left[\stackrel{o}{w}_{4}\right]=-2\right)$, which means that these parameters are irrelevant. To be consistent all irrelevant parameters have to be set to zero. Moreover diagrammatic contributions containing $w_{q}$ do only appear with a prefactor, which is irrelevant. Therefore we do not consider this time ratio as well.

\subsection{Renormalization}

Model H' needs additional renormalization factors, but all of them can be related to the renormalization of model $\mathrm{H}$. In particular we have for the second conserved density $q_{0}$ and the static parameters

$$
\begin{array}{r}
q_{0}=Z_{q}^{1 / 2} q, \quad \stackrel{o}{\tau}-\stackrel{o}{\tau}_{s}=Z_{\phi}^{-1} Z_{\tau} \tau, \\
\stackrel{o}{\tilde{u}}=\mu^{\epsilon} Z_{\phi}^{-2} Z_{\tilde{u}} \tilde{u} A_{d}^{-1}, \quad \stackrel{o}{\gamma_{q}}=\mu^{\epsilon / 2} Z_{\phi}^{-1} Z_{q}^{-1 / 2} Z_{\gamma} \gamma_{q} A_{d}^{-1 / 2} .
\end{array}
$$

One can prove $[23,46]$ the relations

$$
Z_{q}^{-1}=a_{q}^{-1}+\left(\frac{\gamma_{q}}{a_{q}}\right)^{2} A(u), \quad Z_{\gamma}=Z_{q} Z_{r}
$$


$A(u)$ is the additive renormalization constant of the specific heat, calculated within the $\phi^{4}$-model. The parameters $\tilde{u}$ and $\gamma_{q}$ only appear in the combination $u=\tilde{u}-3 \gamma_{q}^{2} / a_{q}$ in $Z_{\phi}$, thus with relations (4.22) follows that all static renormalization factors can be written as function of $u$ and $\gamma_{q}$.

The renormalization of the static vertex functions reads

$$
\Gamma_{\phi \phi}=Z_{\phi} \stackrel{o}{\Gamma}_{\phi \phi}, \quad \Gamma_{q q}=Z_{q} \stackrel{o}{\Gamma}_{q q} .
$$

The Z-factor of the new auxiliary field $\tilde{q}_{0}$

$$
\tilde{q}_{0}=Z_{\tilde{q}}^{-1 / 2} \tilde{q}
$$

is given by $Z_{\tilde{q}}=Z_{q}^{-1}$. The new renormalized OCs are defined as

$$
\stackrel{o}{L}=\mu Z_{L} L \quad, \quad \stackrel{o}{\lambda_{q}}=\mu^{2} Z_{\lambda_{q}} \lambda_{q} \quad, \quad \stackrel{o}{\lambda_{t}}=\mu^{2} Z_{\lambda_{t}} \lambda_{t} .
$$

Using (2.10) and (2.12) the new renormalized dynamic vertex functions are defined as

$$
\Gamma_{q \tilde{q}}=\stackrel{o}{\Gamma}_{q \tilde{q}}, \quad \Gamma_{\phi \tilde{q}}=Z_{\phi}^{1 / 2} Z_{q}^{-1 / 2} \stackrel{o}{\Gamma_{\phi \tilde{q}}}, \quad \Gamma_{q \tilde{\phi}}=Z_{\phi}^{-1 / 2} Z_{q}^{1 / 2} \stackrel{o}{\Gamma}_{q \tilde{\phi}}
$$

From the factorization of the vertex functions into purely static and purely dynamic parts as discussed in the preceding section, follows that the $Z$ factors defined in (4.25) also separate into purely static and purely dynamic parts, namely

$$
Z_{\lambda_{q}}=Z_{q} Z_{\lambda_{q}}^{(d)}, \quad Z_{L}=Z_{\phi}^{1 / 2} Z_{q}^{1 / 2} Z_{L}^{(d)} .
$$

Inserting the separation of the Z-factors and the dynamic vertex functions in static and dynamic contributions in (2.16), (4.26) the renormalization of the purely dynamic functions $f_{\alpha \bar{\beta}}^{(d)}$ are obtained

$$
\stackrel{o}{f_{\phi \tilde{q}}^{(d)}}=Z_{\phi}^{1 / 2} Z_{q}^{1 / 2} f_{\phi \tilde{q}}^{(d)}, \quad \stackrel{o}{f}_{q \tilde{q}}^{(d)}=Z_{q} f_{q \tilde{q}}^{(d)} .
$$

From perturbation theory one can immediately see that $Z_{L}^{(d)}=1$ and $Z_{\lambda_{q}}^{(d)}=1$ in every order of the loop expansion. Thus $L$ and $\lambda_{q}$ stay unrenormalized and merely enter the calculation of the vertex functions as constant parameters. Only $\Gamma$ and $\lambda_{t}$ renormalize, and no new independent renormalization constant is needed. This means that no new critical exponent appears in the asymptotic power laws. This is fundamentally for model $\mathrm{H}$ and model $H^{\prime}$ to belong to the same universality class. With (2.13) and (4.25) the new time ratio $w(4.19)$ renormalizes as

$$
\stackrel{o}{w}=Z_{\Gamma}^{-1 / 2} w
$$

$\ell \frac{d f}{d \ell}=-\frac{1}{2} f\left(\epsilon+\zeta_{\Gamma}^{(d)}[f(\ell), f(\ell) u(\ell), w(\ell]]+\zeta_{\lambda_{t}}[f(\ell), f(\ell) u(\ell), w(\ell)]+\zeta_{\phi}[u(\ell)]\right)$,

$$
\begin{gathered}
\ell \frac{d w}{d \ell}=-\frac{1}{2} w \zeta_{\Gamma}^{(d)}[f(\ell), f(\ell) u(\ell), w(\ell]], \\
\ell \frac{d \Gamma}{d \ell}=\Gamma\left(\zeta_{\Gamma}^{(d)}[f(\ell), f(\ell) u(\ell), w(\ell)]+\zeta_{\phi}[u(\ell)]\right) \quad \ell \frac{d \lambda_{q}}{d \ell}=\lambda_{q}\left(-2+\zeta_{q}\right), \\
\ell \frac{d L}{d \ell}=L\left(-1+\frac{1}{2} \zeta_{\phi}+\frac{1}{2} \zeta_{q}\right) \quad \ell \frac{d \lambda_{t}}{d \ell}=\lambda_{t} \zeta_{\lambda_{t}}[f(\ell), f(\ell) u(\ell), w(\ell)] .
\end{gathered}
$$




\subsection{Temperature dependence of the transport coefficients}

Repeating the steps made in subsection $\mathbf{2 . 3}$ and inserting for the vertex functions we obtain

$$
\begin{gathered}
D(\ell)=(\mu \ell)^{2} \hat{\Gamma}_{\phi \phi}[u(\ell)] \Gamma(\ell)(1+G[w(\ell), f(\ell), f(\ell) u(\ell)]), \\
\frac{k_{T}(\ell)}{T}=\frac{\rho \stackrel{o}{L}}{R T D(\ell)}, \\
\frac{\kappa(\ell)}{\rho T}=\frac{(\mu \ell)^{4}}{D(\ell)}\left(\frac{\partial \sigma}{\partial T}\right)_{c, P} \hat{\Gamma}_{\phi \phi}[u(\ell)] \hat{\Gamma}_{q q}\left[u(\ell), \gamma_{q}(\ell)\right] \\
\left.\times\left\{\lambda_{q}(\ell) \Gamma(\ell)(1+G[w(\ell), f(\ell), f(\ell) u(\ell)])-L^{2}(\ell)\right]\right\}, \\
\bar{\eta}(\ell)=\frac{1}{R T}(\mu \ell)^{2} \lambda_{t}(\ell)(1+E[w(\ell), f(\ell), f(\ell) u(\ell)]) .
\end{gathered}
$$

In the same way as before for pure fluids, using the matching condition (2.24) and approximating $\zeta_{\phi}$ by $\eta$, we obtain the final results for the TC's, namely the mass diffusion

$$
D(t)=\xi_{0}^{-\eta} \xi(t)^{-2+\eta} \hat{\Gamma}_{\phi \phi}\left(u^{*}\right) \Gamma^{(d)}(t)(1+G[w(t), f(t), f(t) u(t)])
$$

the shear viscosity

$$
\bar{\eta}(t)=A_{d} k_{B} T \xi_{0}^{\eta} \xi(t)^{\epsilon-\eta} \frac{1+E[w(t), f(t), f(t) u(t)]}{\Gamma^{(d)}(t) f^{2}(t)}
$$

and the thermal conductivity

$$
\frac{\kappa(t)}{\rho T}=\frac{\rho}{R T} \stackrel{o}{\lambda}_{q}\left(1-\frac{w^{2}(t)}{1+G[w(t), f(t), f(t) u(t)]}\right) .
$$

Thus the critical enhancement of the thermal conductivity is directly related to the flow of the parameter $w$. Unfortunately we are not aware of any measurements of the thermal conductivity near the consolute point. The thermal diffusion ratio is exactly the inverse of the mass diffusion $k_{T}(t)=$ $\frac{\rho_{L}^{\circ}}{R D(t)}$. This has been verified in [47] for aniline-cyclohexane up to values of $t \sim 10^{-2}$, but it would be worthwhile to prove this further out to the background region.

\subsection{Nonuniversal Kawasaki amplitude}

The corresponding Kawasaki amplitude [30] at the consolute point reads (at $d=3)$

$$
R_{e x p}^{c o n s}=\frac{6 \pi \eta D \xi}{k_{B} T}
$$


Inserting the theoretical expressions we obtain the nonasymptotic amplitude

$$
R_{t h}^{c o n s}=\frac{3}{2} \hat{\Gamma}_{\phi \phi}(u(\ell)) \frac{(1+G[w(\ell), f(\ell), f(\ell) u(\ell)])(1+E[w(\ell), f(\ell), f(\ell) u(\ell)])}{f^{2}(\ell)} .
$$

Although the asymptotic value of the Kawasaki amplitude is the same as in the pure liquid, the nonasymptoic expression is different. We would like to mention that it is the same as for the plait point. However the definition of the Kawasaki amplitude by the experimental quantities is different for the plait point (see [15]).

\section{Comparison with experiment in mixtures}

The general procedure is the same as already described, however we have now more parameters to determine. We use again the shear viscosity in order to find these parameters, but one has to keep in mind that the accuracy of the flow found depends on fine details of the shear viscosity data. The thermal conductivity is most sensitive to the flow of $w$ and would be the primary experimental quantity to be fitted in order to find $w\left(t_{0}\right)$. Unfortunately no such data are available and we therefore use the shear viscosity for the determination of all three initial parameters, $\Gamma^{(d)}\left(t_{0}\right), f\left(t_{0}\right)$ and $w\left(t_{0}\right)$.

Comparison with aniline-cyclohexane We use the shear viscosity data of [48] and the mass diffusion from [47]. No correction has been made for the regular temperature dependence. In the background the shear viscosity is decreasing since we approach the phase transition from above $T_{c}$. The correction procedure would lead to an increase of the shear viscosity values. This may lead to smaller $w$ values in the background predicting a smaller critical enhancement of the thermal conductivity.

Comparison with butoxyethanol-water. We use the data of the shear viscosity, the mass diffusion (represented by $D=5.6810^{-6} t^{-0.692}$ ) and the correlation length (the asymptotic fit) measured in [49]. We have performed fits of the shear viscosity with and without correction of the regular temperature behaviour (see Table 3). In the background the shear viscosity is increasing since we approach $T_{c}$ from below. This leads to a decrease for the corrected data. The interesting point is that the best fit of the corrected data leads to a nonzero background value of the parameter $w$ indicating a critical enhancement of the thermal conductivity.

\begin{tabular}{|c|c|c|c|c|c|c|c|}
\hline Systems & $\xi_{0}[\AA]$ & $T_{c}[K]$ & $t_{0} 10^{2}$ & $t_{1} 10^{4}$ & $\Gamma^{(d)} 10^{18}\left[\mathrm{~cm}^{4} / \mathrm{s}\right]$ & $f$ & $w$ \\
\hline${ }^{3} \mathrm{He}$ & 2.7 & 3.3086 & 10 & 1 & 2.105 & 0.345 & - \\
${ }^{4} \mathrm{He}$ & 2.0 & 5.1895 & 10 & 1 & 0.833 & 0.495 & - \\
$\mathrm{C}_{2} \mathrm{H}_{6}$ & 1.9 & 305.33 & 10 & 5 & 3.867 & 0.576 & - \\
$\mathrm{A}-\mathrm{C}$ & 2.4 & 303 & 3 & 1 & 0.05352 & 1.075 & 0.897 \\
$2 \mathrm{~B}-\mathrm{W}$ & 4.2 & 322.53 & 0.7 & 0.1 & 0.2051 & 1.015 & 0 \\
$2 \mathrm{~B}-\mathrm{W}$ & 4.2 & 322.53 & 0.7 & 0.1 & 0.17 & 1.124 & 0.399 \\
$2 \mathrm{~B}-\mathrm{W}$ & 4.2 & 322.53 & 1 & 0.1 & 0.1375 & 1.124 & 0.402 \\
\hline
\end{tabular}

Table 3. Parameters used and found for pure fluids and mixtures 


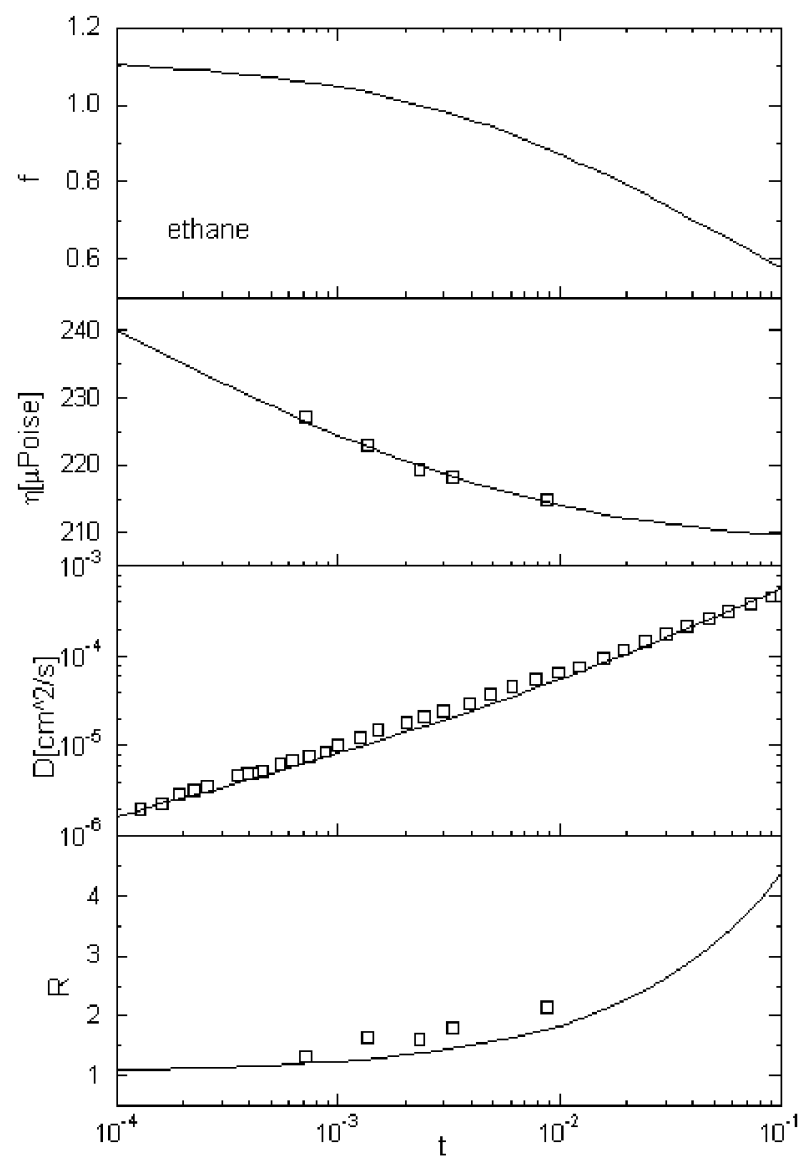

Figure 3. Comparison of theory with ethane data (see text for the Refs.). We show the flow of $f$ found from a fit of the shear viscosity $\eta$ and compare with the prediction of the thermal diffusivity $D_{T}$. We also compare with the Kawasaki amplitude $R$.

\section{Outlook}

There seems to be a remarkable difference between the behaviour of the renormalized mode coupling constant $f(t)$ in pure fluids and mixtures at the consolute point. Whereas in pure fluids $f\left(10^{-2}\right)$ is clearly smaller than its fixed point value, this is not the case at the consolute point (we have seen this also from fits of the shear viscosity of other mixtures). In consequence the mass diffusion shows almost the asymptotic behaviour. A crucial point however is the flow of the parameter $w(t)$, which determines the behaviour of the thermal conductivity at zero mass flow. The size of it's critical enhancement is an important test of the $w$-flow found from the shear viscosity 
alone. The larger the enhancement the larger the background value of $w$. Unfortunately we do not know any quantitative or even qualitative measurements of the thermal conductivity in mixtures at the consolute point.
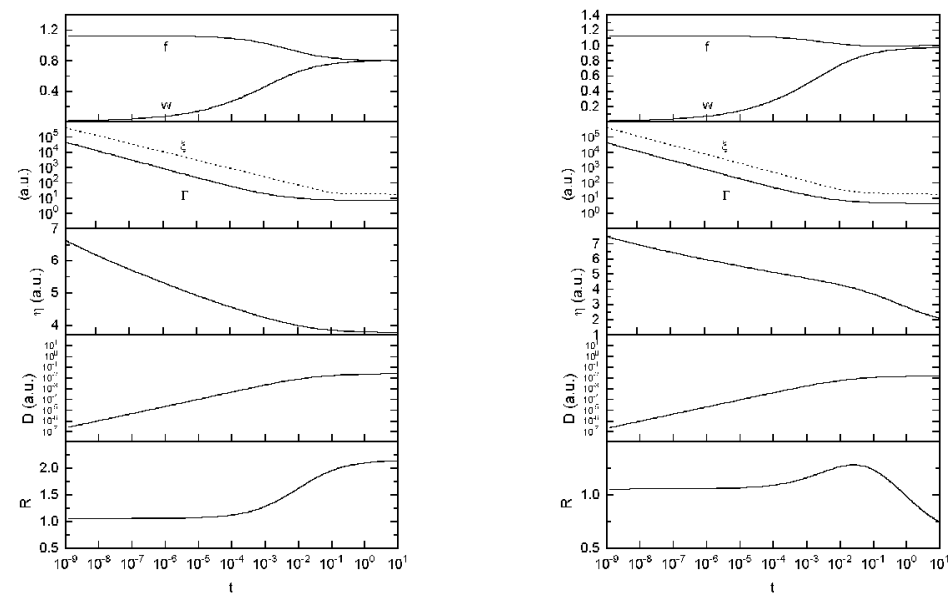

Figure 4. Temperature dependence of (a) the mode-coupling $f$ and ratio $w$ according to the flow equation (4.30), and (4.31) (b) the correlation length $\xi$ and the order parameter diffusion rate $\Gamma$ according to the flow equation (4.32), (c) the shear viscosity $\bar{\eta}$ according to equation (4.37), (d) the thermal diffusivity $D$ according to equation (4.34), and the (e) Kawasaki amplitude ratio $R$ according to equation (4.42) in one loop order (Appendix A). All quantities reach temperature independent background values, $f, w$ and $R$ reach at $T_{c}$ their one loop fixed point values $f^{*}, w^{*}=0$ and $R^{*}=1.056$.

Our analysis might be applicable in other system too e.g. (i) ionic fluids and mixtures [50] and/or (ii) polymeric blends [51].

From the flow of $w$ and $f$ (see Figure 6) one may calculate according to equation (4.40) the critical enhancement of the thermal conductivity of the 2-Butoyethanol-Water-mixture. We find over the interval of $t=10^{-2}$ to $t=$ $10^{-3}$ an increase of the thermal conductivity of $15 \%$. This compares quite well with measurements made by $\mathrm{H}$. Mensah-Brown and W. A. Wakeham (we estimate a $6.5 \%$ increase from their experimental results). See the Refs. H. Mensah-Brown and W. A. Wakeham, Thermal Conductivity of a Liquid Mixture Showing a Lower Critical Solution Temperature, Int. J. Thermophys. 15, 647 (1994) and Thermal Conductivity of Water and 2-nButoxyethanol and Their Mixture in the Temperature Range 305-350 K at Pressures up to $150 \mathrm{MPa}$, Int. J. Thermophys. 16, 237 (1995). We thank J. V. Sengers for pointing to this reference.

\section{Acknowledgements}

We thank H. Meyer, J. Sengers, and W. Schröer for helpful discussions. 


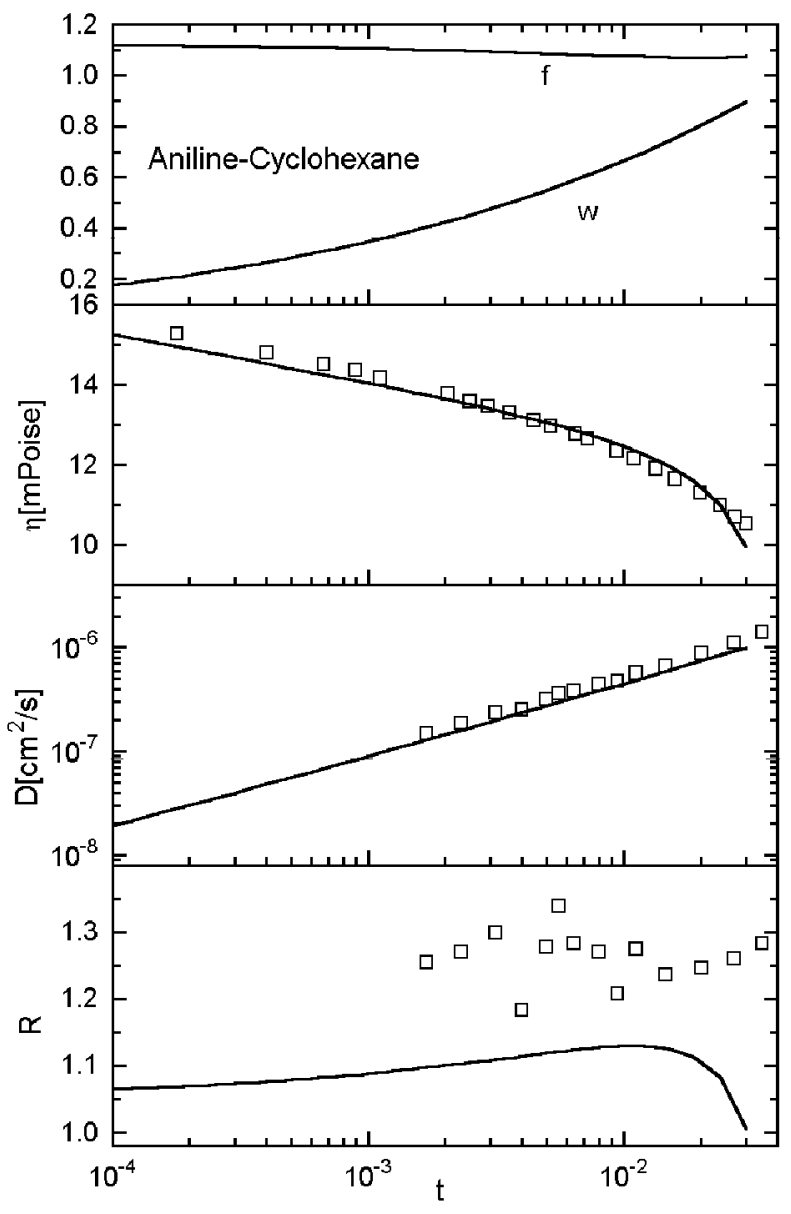

Figure 5. Comparison of theory with Aniline-Cyclohexane data (see text for the Refs.). We show the flow of $f$ and $w$ found from a fit of the shear viscosity $\eta$ and compare our prediction with the mass diffusivity $D_{T}$. We also compare with the Kawasaki amplitude $R$. 


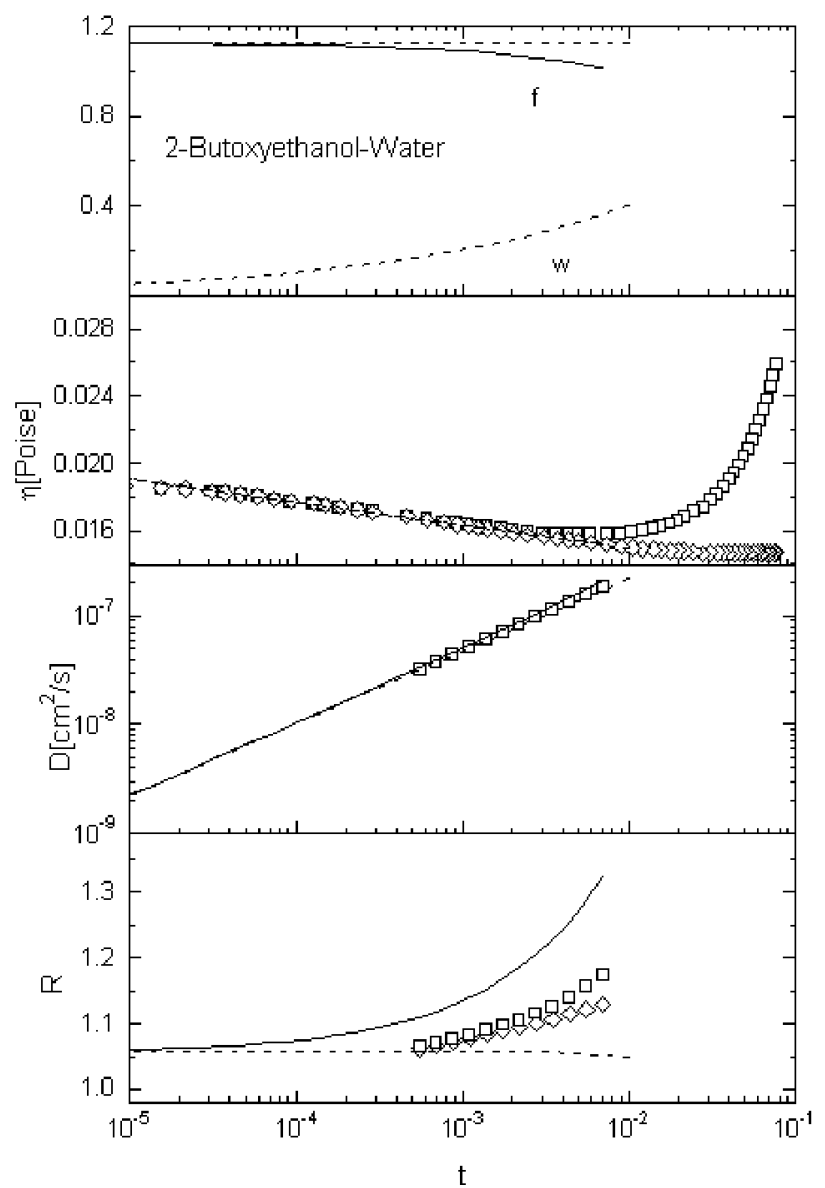

Figure 6. Comparison of theory with 2-Butoxyethanol-Water data (see text for the Refs.). We show the flow of $f$ and $w$ found from a fit of the shear viscosity $\eta$ and compare our prediction with the mass diffusivity $D_{T}$. The solid curves result of a fit of the uncorrected shear viscosity data (open squares) and the dashed curves from the shear viscosity data corrected for the regular temperature dependence (open diamonds). We also compare with the respective Kawasaki amplitudes $R$. 


\section{Appendices}

\section{A Explicit results in one loop order for pure fluids}

The static $\zeta$-functions in one loop order are

$$
\zeta_{\phi}=0, \quad \zeta_{r}=\frac{1}{2} u, \quad \zeta_{u}=\frac{3}{2} u
$$

and the dynamical $\zeta$-functions

$$
\zeta_{\Gamma}^{(d)}=-\frac{3}{4} f^{2}, \quad \zeta_{\lambda_{t}}=-\frac{1}{24} f^{2} .
$$

The static vertex function is $\hat{\Gamma}_{\phi \phi}=1$. The dynamic vertex functions in this order are found to be

$$
\begin{aligned}
& \stackrel{o(d)}{f_{\phi \tilde{\phi}}}=\stackrel{o}{\Gamma}\left(1-\frac{\stackrel{o}{f}^{2}}{\stackrel{o}{r}} \int_{k^{\prime}} \frac{\sin ^{2} \theta}{\stackrel{o}{r}+k^{\prime 2}}\right), \\
& \stackrel{o}{f_{t \tilde{t}}(d)}=\stackrel{o}{\lambda_{t}}\left\{1+\frac{o^{2}}{6}\left[\int_{k^{\prime}} \frac{\sin ^{2} \theta}{\left(r+o+k^{\prime 2}\right)^{2}}-2 \int_{k^{\prime}} \frac{\sin ^{2} \theta \cos ^{2} \theta}{\left(r+k^{\prime 2}\right)^{2}}\left(1+\frac{k^{\prime 2}}{r+k^{\prime 2}}\right)\right]\right\} .
\end{aligned}
$$

The $\epsilon$-expanded amplitude functions $G$ and $E$ are in one loop order

$$
G=-\frac{1}{16} f^{2}(\ell) \quad E=-\frac{1}{36} f^{2}(\ell) .
$$

\section{B Solutions of flow equations for pure fluids}

The one loop flow equations (2.22),(2.33) may be solved analytically. We find for the dimensionless mode coupling constant

$$
f^{2}(t)=\frac{24}{19}\left[1+\frac{\xi\left(t_{0}\right)}{\xi(t)}\left(\frac{24}{19 f_{0}^{2}}-1\right)\right]
$$

and for $\Gamma^{(d)}$

$$
\Gamma^{(d)}(t)=\Gamma_{0}^{(d)}\left(\frac{19 f_{0}^{2} \xi(t)}{24 \xi\left(t_{0}\right)}\left[1+\frac{\xi\left(t_{0}\right)}{\xi(t)}\left(\frac{24}{19 f_{0}^{2}}-1\right)\right]\right)^{\frac{18}{19}}
$$

with the initial values $f_{0}<\frac{24}{19}$ and $\Gamma_{0}^{(d)}$ at $t_{0}$.

\section{One loop $\zeta$-functions for mixtures}

The static $\zeta$-functions are the same as above and the dynamical $\zeta$-functions read

$$
\zeta_{\Gamma}^{(d)}=-\frac{3}{4} f^{2}, \quad \zeta_{\lambda_{t}}=-\frac{1}{24} \frac{f^{2}}{1-w^{2}} .
$$

The static vertex function is $\hat{\Gamma}_{\phi \phi}=1$. The dynamic vertex functions in this order are found to be

$$
\begin{aligned}
& \stackrel{o(d)}{f_{q \tilde{q}}^{(d)}}=\stackrel{o}{\mu}, \quad \stackrel{o(d)}{f_{\phi \tilde{q}}}=\stackrel{o(d)}{f_{q \tilde{\phi}}}=\stackrel{o}{L}, \\
& f_{\phi \tilde{\phi}}^{o(d)}=\stackrel{o}{\Gamma}\left(1-\frac{\stackrel{o}{f}}{\stackrel{o}{r}} \int_{k^{\prime}} \frac{\sin ^{2} \theta}{\stackrel{o}{r}+k^{2}}\right),
\end{aligned}
$$




$$
\stackrel{o(d)}{f_{t \tilde{t}}}=\stackrel{o}{\lambda} t\left\{1+\frac{o^{2}}{6(1-\stackrel{o}{w})}\left[\int_{k^{\prime}} \frac{\sin ^{2} \theta}{\left(\stackrel{o}{r}+k^{\prime 2}\right)^{2}}-2 \int_{k^{\prime}} \frac{\sin ^{2} \theta \cos ^{2} \theta}{\left(\stackrel{o}{r}+k^{\prime 2}\right)^{2}}\left(1+\frac{k^{\prime 2}}{r+k^{\prime 2}}\right)\right]\right\} .
$$

The $\epsilon$-expanded amplitude functions $G$ and $E$ are in one loop order

$$
G=-\frac{1}{16} f^{2}(\ell) \quad E=-\frac{1}{36} \frac{f^{2}(\ell)}{1-w^{2}(\ell)}
$$

\section{References}

[1] Privman V., Hohenberg P.C., Aharony A. Universal critical-point amplitude relations in: Phase transitions and critical phenomena, vol. 14. Eds. C.Domb, J.L.Lebowitz. Academic Press, 1991.

[2] Beysens D. Status of the experimental situation in critical binary fluids in: Phase transition, status of the experimental and theoretical situation, Cargese 1980, ed. by M.Levy, J.C.Le Guillou and J.Zinn-Justin. Plenum Press, 1981.

[3] Beysens D., Paladin G., Bourgou A. Dynamic corrections to transport coefficients of critical binary fluids. // J. Physique Lettres, 1983, vol. 44, p. L-649.

[4] Beysens D., Bourgou A., Paladin G. Experimental determination of universal amplitude combination for binary fluids. II. Dynamics. // Phys. Rev. A, 1984, vol. 30 , p. 2686.

[5] Dohm V., Folk R. Nonlinear dynamic renormalization-group analysis above and below the superfluid transition in ${ }^{4}$ He. // Phys. Rev. Lett., 1981, vol. 46, p. 349.

[6] Dohm V., Folk R. Critical dynamics near the $\lambda$-transition of ${ }^{3} \mathrm{He}^{4} \mathrm{He}$ mixtures. // Phys. Rev. B, 1983, vol. 28, p. 1332; Moser G., Folk R. Critical dynamics in the normal phase near the $\lambda$-transition in ${ }^{3} \mathrm{He}-{ }^{4} \mathrm{He}$ mixtures I: Theoretical results, II: Comparison with experiment. // J. Low. Temp. Phys., 1992, vol. 86, p. 57, 99 .

[7] Dohm V. Renormalization-group theory of critical phenomena near the lambda transition of ${ }^{4}$ He. // J. Low. Temp. Phys., 1987, vol. 69, p. 51.

[8] Dohm V. The superfluid transition in confined ${ }^{4}$ He: Renormalization-group theory. // Physica Scripta T, 1993, vol. 49, p. 49.

[9] Dohm V. The superfluid transition of ${ }^{4} \mathrm{He}$ in the presence of a heat current: renormalization-group theory. // Physica B, 1994, vol. 197, p. 215.

[10] Sengers J.V. Effects of critical fluctuations on the thermodynamic and transport properties of supercritical fluids in: Supercritical fluids: fundamentals for application, E. Kiran and J. M. H. Levelt Sengers, eds. Kluwer, Dordrecht, 1994.

[11] Ferrell R.A. Decoupled-mode dynamical scaling theory of the binary-liquid phase transition. // Phys. Rev. Lett., 1970, vol. 24, p. 1169; Perl R., Ferrell R.A. Decoupled-mode theory of critical viscosity and diffusion in the binary-liquid phase transition. // Phys. Rev. A, 1972, vol. 6, p. 2358.

[12] Kiselev S.B., Kulikov V.D. Crossover behaviour of the transport coefficients of critical binary mixtures. // Int. J. Thermophys., 1994, vol. 15, p. 283.

[13] Siggia E.D., Halperin B.I., Hohenberg P.C. Renormalization-group treatment of the critical dynamics of the binary-fluid and gas-liquid transition. // Phys. Rev. B, 1976, vol. 13, p. 2110.

[14] Folk R., Moser G. Critical dynamics near the liquid-vapour transition in mixtures. // Europhys. Lett., 1993, vol. 24, p. 533.

[15] Folk R., Moser G. Critical dynamics near plait points in mixtures. // J. Low Temp. Phys., 1995, vol. 99, p. 11.

[16] Folk R., Moser G. Universality versus nonuniversality of critical transport properties in liquid mixtures. // Int. J. Thermophys., to be published (1995).

[17] Folk R., Moser G. Nonuniversal dynamical crossover in pure and binary fluids near a critical point. // Phys. Rev. Lett., 1995, vol. 75, p. 2706. 
[18] Luettmer-Strathman J. Transport properties of fluids and fluid mixtures in the critical region: Thesis University of Maryland, 1994.

[19] Olchowy G.A., Sengers J.V. Crossover from singular to regular behaviour of the transport properties of fluids in the critical region. // Phys. Rev. Lett., 1988, vol. 61, p. 15.

[20] Kawasaki K. Mode coupling and critical dynamics in: Phase transitions and critical phenomena, vol.5a, eds. C.Domb, M.S.Green. Academic Press, 1976.

[21] Luettmer-Strathman J., Sengers J.V., Olchowy G.A. Non-asymptotic critical behaviour of the transport properties of fluids. // J. Chem. Phys., in press (1995).

[22] Luettmer-Strathman J., Sengers J.V. The transport properties of fluid mixtures near the vapour-liquid critical line. Preprint, August 1995.

[23] De Dominicis C., Peliti L. Field-theory renormalization and critical dynamics above $T_{c}$ : Helium, antiferromagnets and liquid-gas systems. // Phys. Rev. B, 1978 , vol. 18 , p. 353 .

[24] Paladin G., Peliti L. Fixed dimensional computation of critical transport properties of fluids. // J. Physique Lett., 1982, vol. 43, p. 15; Erratum 1984, vol. 45 , p. 289.

[25] Amit D.J. Field theory, the renormalization group and critical phenomena, $2^{\text {nd }}$ ed. World Scientific, 1984.

[26] Schloms R., Dohm V. Minimal renormalization without $\epsilon$-expansion: Critical behaviour in three dimensions. // Nucl. Phys. B, 1989, vol. 328, p. 639.

[27] In [15] the condition $r(\ell)=(\mu \ell)^{2}$ was used (equation (128) there). This leeds to the additional factor $\frac{\hat{\Gamma}_{\phi \phi}^{\prime}[u(\ell)]}{\hat{\Gamma}_{\phi \phi}[u(\ell)]}$ in the calculation of the correlation length (equation (168) there) and in the dynamic amplitude (equation (170)) there). This factor is absent with the matching condition (2.24) (see the discussion in $[26])$.

[28] Dohm V., Folk R. Nonasymptotic critical dynamics near the superfluid transition in ${ }^{4}$ He. // Z. Physik B, 1980, vol. 40, p. 79.

[29] Dohm V. General relation between the specific heat above and below a secondorder phase transition. // Phys. Rev. Lett., 1984, vol. 53, p. 1379, 2520.

[30] Kawasaki K. Kinetic equations and time correlation functions of critical fluctuations. // Ann. Phys., 1970, vol. 61, p. 1.

[31] Sengers J.V., Levelt-Sengers J.M.H. Critical phenomena in classical fluids in: Progress in liquid physics, ed. C.A.Croxton. Wiley, 1987.

[32] Pittman E., Dorion T., Meyer H. Equation of state and critical exponents of ${ }^{3} \mathrm{He}$ and $\mathrm{a}^{3} \mathrm{He}^{4} \mathrm{He}$ mixture near their liquid-vapour critical point. // Phys. Rev. B, 1979, vol. 20, p. 3678 .

[33] Behringer R.P., Doiron T., Meyer H. Equation of state of ${ }^{3}$ He near its liquidvapour critical point. // J. Low Temp. Phys., 1976, vol. 24, p. 315.

[34] Brown G.R., Meyer H. Study of the specific-heat singularity of $\mathrm{He}^{3}$ near its critical point. // Phys. Rev. A, 1972, vol. 6, p. 364.

[35] Agosta C.C., Wang S., Cohen L.H., Meyer H. Transport properties of helium near the liquid-vapour critical point. IV. The shear viscosity of ${ }^{3} \mathrm{He}$ and ${ }^{4} \mathrm{He}$. // J. Low. Temp. Phys., 1987, vol. 67, p. 237.

[36] Pittman E., Cohen L.H., Meyer H. Transport properties of helium near the liquid-vapour critical point. I. Thermal conductivity of ${ }^{3}$ He. // J. Low. Temp. Phys., 1982, vol. 46, p. 115.

[37] Acton A., Kellner K. The low temperature thermal conductivity of ${ }^{4} \mathrm{He}$, I. Measurements between 3.3 and $20 \mathrm{~K}$ on the dilute gas, the dense gas and the liquid. // Physica, 1977, vol. 90B, p. 192.

[38] Roach P.R. Pressure-density-temperature surface of $\mathrm{He}^{4}$ near the critical point. // Phys. Rev., 1968, vol. 170, p. 213. We used $\gamma=1.18$ in our fit of their data.

[39] Kierstead H.A. PTV surface of $\mathrm{He}^{4}$ near its critical point. // Phys. Rev. A, 1973, vol. 7, p. 242 . 
[40] Moldover M.R. Scaling of the specific-heat singularity of $\mathrm{He}^{4}$ near its critical point. // Phys. Rev., 1969, vol. 182, p. 342.

[41] Tominaga A. Intensity of critical opalescence of helium-4. // J. Low Temp. Phys., 1974, vol. 16, p. 571.

[42] Iwasaki H., Takahashi M. Viscosity of carbon dioxide and ethane. // J. Chem. Phys., 1981, vol. 74, p. 1930.

[43] Mostert R., van den Berg H.B., van der Gulik P.S., Sengers J.V. The thermal conductivity of ethane in the critical region. // J. Chem. Phys., 1990, vol. 92, p. 5454; Mostert R. Ph. D. Thesis. Van der Waals-Zeeman Laboratory, Amsterdam, 1991.

[44] Jany P., Straub J. Thermal diffusivity of fluids in a broad region around the critical point. // Int. J. Thermophys., 1987, vol. 12, p. 165.

[45] Roe D.B., Meyer H. Ultrasonic dispersion and attenuation near the liquid-gas critical point of ${ }^{3}$ He. // J. Low Temp. Phys., 1978, vol. 30, p. 91; Roe D.B., Wallace B.A., Meyer H. Ultrasonic dispersion and attenuation near the liquidgas critical point of ${ }^{4}$ He. // J. Low Temp. Phys., 1974, vol. 16, p. 51.

[46] Dohm V. Nonuniversal critical phenomena along the lambda line of ${ }^{4} \mathrm{He}, \mathrm{I}$. Specific heat in three dimensions. // Z. Phys. B, 1985, vol. 60, p. 61.

[47] Giglio M., Vendramini A. Thermal-diffusion measurements near a consolute critical point. // Phys. Rev. Lett., 1975, vol. 35, p. 561.

[48] Arcovito G., Faloci C., Roberti M., Mitsura L. Shear viscosity of the binary system aniline-cyclohexane near the critical point. // Phys. Rev. Lett., 1969, vol. 22, p. 1040.

[49] Zielesny A., Schmitz J., Limberg S., Aizpiri A.G., Fusenig S., Woermann D. Viscosity and diffusivity of a binary liquid mixture of critical composition: Study of the system 2-butoxyethanol/water. // Int. J. Thermophys., 1994, vol. 15 , p. 67.

[50] Schröer W., Wiegand S., Kleemeier M., Weingärtner H. Critical fluctuations in electrolytes with a liquid-liquid phase transition. // J. Phys. Cond. Matter, 1994, vol. 6, p. A157; Schröer W. private communication.

[51] Theobald W., Meir G. Static and dynamic critical behaviour of a binary polymer blend in the strong fluctuation limit: A light scattering study. // Phys. Rev. E, 1995, vol. 51, p. 5776 and Refs. therein.

\title{
КОЕФІЩІЕНТИ ПЕРЕНОСУ В ЧИСТИХ РІДИНАХ ТА СУМІШАХ ПОБЛИЗУ КРИТИЧНОЇ ТОЧКИ: ПОРІВНЯННЯ РЕЗУЛЬТАТІВ ОДНОПЕТЛЕВОГО НАБЛИЖЕННЯ 3 ЕКСПЕРИМЕНТОМ
}

\author{
Р.Фолк, Г.Мозер
}

Проводиться огляд залежності від неасимптотичної критичної температури коефіцієнтів переносу в чистих рідинах поблизу точки фазового переходу та в сумішах поблизу точки розшарування розрахованих у найнижчому нетривіальному наближенні ренормалізаційної теорії груп. Два динамічних фонових параметри теорії (відповідно три для суміші) фіксується при отриманні одного з коефіцієнтів переносу (тут взято зсувну в'язкість). Тоді інші коефіцієнти переносу завбачаються без жодних підгоночних параметрів. Запропонований аналіз показує добру узгодженість 3 асимптотичним однопетлевим значенням амплітуди Кавасакі $R=1,056$. Відхилення коефіцієнтів переносу в асимптотичній області відбувається із-за однопетлевого наближення для асимптотичних експонент. 